\title{
Ações de enfermagem junto aos portadores de diabetes mellitus residentes da zona
}

\section{rural}

Nursing actions with diabetes mellitus patients living in rural areas

Acciones de enfermería con pacientes con diabetes mellitus que viven en zonas rurales

Recebido: 10/05/2021 | Revisado: 16/05/2021 | Aceito: 17/05/2021 | Publicado: 04/06/2021

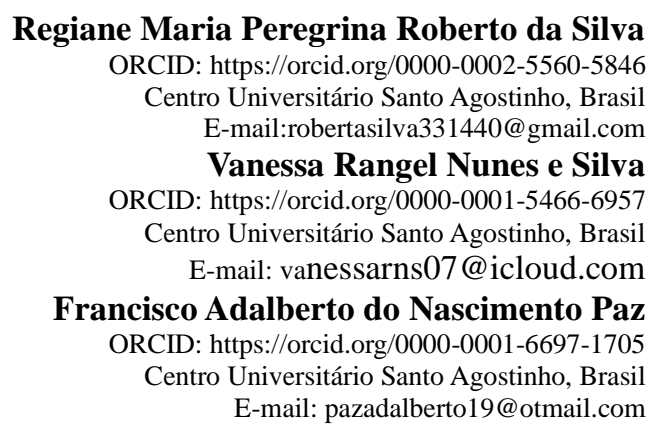

\begin{abstract}
Resumo
A assistência de enfermagem é crucial no que se refere ao cuidado do paciente acometido pelo diabetes mellitus, bem como o desenvolvimento de ações, principalmente àqueles que residem em ambiente rural por apresentarem um estilo de vida particular, além disso, enfrentam dificuldades no acesso ao serviço de saúde ou acessibilidade reduzida devido a não residir próximo a esses institutos. O objetivo deste estudo foi investigar as publicações que tratam das ações de enfermagem realizadas junto aos portadores de diabetes mellitus residentes da zona rural, com destaque aos aspectos relevantes apontados por estes profissionais enfermeiros que atuam na assistência dessas pessoas, conforme as publicações científicas. Utilizou-se a revisão integrativa, em que 10 artigos científicos disponibilizados na íntegra, nos últimos 5 anos, selecionados na Biblioteca Virtual de Saúde, compuseram a amostra analisada. Os resultados demonstraram a relevância do desenvolvimento das ações do profissional enfermeiro frente aos pacientes acometidos pelo diabetes mellitus e os resultados positivos destas, mostram que a aplicabilidade e aperfeiçoamento dessas ações que podem ser desenvolvidas pelo profissional enfermeiro são de total relevância, tanto para a prevenção, como para a lida com a doença depois de diagnosticada, principalmente no cenário rural onde ficou evidenciada a necessidade de um olhar mais especial para estes.
\end{abstract}

Palavras-chave: Diabetes mellitus; Enfermagem; Zona rural.

\begin{abstract}
Nursing care is crucial when it comes to the care of the patient affected by diabetes mellitus, as well as the development of actions, especially for those who live in rural areas because they present a particular lifestyle, in addition, they face difficulties in accessing the health service or reduced accessibility due to not living near these institutions. The aim of this study was to investigate the publications that deal with nursing actions performed with diabetes mellitus patients living in rural areas, highlighting the relevant aspects pointed out by these nursing professionals who work in the care of these people, according to scientific publications. The integrative review was used, in which 10 scientific articles available in full, in the last 5 years, selected from the Virtual Health Library, composed the analyzed sample. The results showed the relevance of the development of actions of the professional nurse in front of patients affected by diabetes mellitus and the positive results of these, show that the applicability and improvement of these actions that can be developed by professional nurses are of total relevance, both for prevention, as for dealing with the disease after it is diagnosed, especially in the rural setting where it was evidenced the need for a more special look for them.
\end{abstract}

Keywords: Diabetes Mellitus; Nursing; Rural area.

\section{Resumen}

La asistencia de enfermería es crucial en lo que se refiere a los cuidados del paciente afectado por la diabetes mellitus, así como al desarrollo de acciones, especialmente para aquellos que residen en zonas rurales por presentar un estilo de vida particular, además, se enfrentan a dificultades de acceso al servicio sanitario o a una accesibilidad reducida por no residir cerca de estos institutos. El objetivo de este estudio fue investigar las publicaciones que abordan las acciones de enfermería realizadas con pacientes con diabetes mellitus que viven en zonas rurales, destacando los 
aspectos relevantes señalados por estos profesionales de enfermería que trabajan en el cuidado de estas personas, según las publicaciones científicas. Se utilizó la revisión integradora, en la que 10 artículos científicos disponibles en el íntegro, en los últimos 5 años, seleccionados en la Biblioteca Virtual de Salud, componen la muestra analizada. Los resultados mostraron la relevancia del desarrollo de acciones profesionales de enfermería para los pacientes afectados por la diabetes mellitus y los resultados positivos de las mismas, demuestran que la aplicabilidad y mejora de estas acciones que pueden desarrollar los profesionales de enfermería son de total relevancia, tanto para la prevención como para el abordaje de la enfermedad una vez diagnosticada, especialmente en el ámbito rural donde se evidenció la necesidad de una mirada más especial para ellos.

Palabras clave: Diabetes mellitus; Enfermería; Zona rural.

\section{Introdução}

O Diabetes Mellitus (DM), geralmente é causada pela resistência ou pela secreção insuficiente de insulina no sangue. O hormônio em questão, é fabricado pelas células betas nas ilhotas de Langherans no pâncreas. O DM declara-se como um fator no aumento da morbimortalidade entre a população, com uma significativa perda da qualidade de vida das pessoas acometidas com a patologia. É uma das principais causas de insuficiência renal, amputação de membros inferiores, cegueira e doença cardiovascular. Ainda segundo os autores está problemática torna-se ainda mais complicada para pessoas acometidas com DM residentes na Zona Rural, por apresentar dificuldades no acesso ao serviço de saúde ou acessibilidade reduzida devido a não residir próximo a esses institutos (Valentim; Haddad; Rossaneis, 2015).

A dificuldade de acesso e a acessibilidade aos serviços de saúde tem um déficit na prática, como a desigualdade, inadequação e a baixa qualidade dos serviços, além da ausência de atenção integral que não atendem as necessidades de qualidade de vida da população. (Bastista; Caldas, 2017).

Os comportamentos influenciam nas mudanças de atitudes e na forma como as pessoas lidam com a situação, entretanto vários aspectos de suas vidas dependerão das imagens de velhice. O processo de envelhecimento é influenciado pela cultura e pelas condições da vida que gera inúmeras situações de dependência que são assumidas pelas famílias. Várias ações educativas podem ser desenvolvidas para possível transformação e ruptura de dogmas sociais, facilitando a adesão do usuário idoso aos serviços de saúde (Batista, Caldas, 2017).

Se compararmos o idoso diabético ao não diabético e possível observar que o portador de DM está mais sujeito a desenvolver incontinência urinária, depressão, como também ser mais medicado e apresentar dores crônicas, o que favorece o surgimento de limitações tanto cognitivas como funcionais que causam dependência e carência por cuidados especiais, afetando a dinâmica familiar (Sousa et al., 2021).

Lembrado que pessoas acometidas pelo diabetes residentes das áreas rurais geralmente alimentam-se de suas próprias plantações e agricultura familiar o que deixa claro a necessidade de uma abordagem diferenciada quanto às terapêuticas que solicitam determinado estilo de vida como por exemplo com relação a efetuar dieta alimentar (Farias et al., 2016).

Quando se trata de educação em saúde o estabelecimento de uma rede de apoio apresenta-se como um excelente fator para alcançar sucesso no processo educativo. Visto isso fica nítida a importância da promoção de atividades educativas em grupo visando o esclarecimento de informações e trocas de experiências, envolvendo também a família dos diabéticos os tornando aliados capacitados para a eficácia do tratamento e cuidados. Contudo faz-se necessária a continuidade destas ações educativas nos serviços de saúde para que seja realizada uma assistência de maneira eficaz e duradoura (Zanetti et al., 2015).

Com isso, faz-se necessário a elaboração de métodos educativos que permitam que as pessoas com diabetes mellitus, possam além de adquirir conhecimento sobre o tratamento da doença, possa também utilizá-la no seu dia a dia. Portanto, tornase indispensável que o profissional veja o paciente de maneira holística, inserida em um contexto social e cultural para que medidas de prevenção primária e secundária sejam estabelecidas, além do desenvolvimento da atenção primária à pessoa com DM (Valentim; Haddad; Rossaneis, 2015). 
No âmbito Estratégia Saúde da Família no que se refere ao diabetes mellitus, tem-se a educação em saúde como um primordial elemento de prevenção e promoção de saúde, porém é sabido que para alcançar resultados eficazes, as ações devem visar não apenas a troca de informações, mas também, em orientações capazes de formar uma consciência saudável e capaz de promover o autocuidado dessa população (Brasil, 2012).

A enfermagem inserida na atenção básica tem papel importante nas ações que visam a promoção de saúde e prevenção de doenças, destacando-se nesse âmbito, além de estar no primeiro nível de atendimento. É importante que o indivíduo com diabetes e seus familiares possuam conhecimento a respeito de todos os cuidados que são necessários, como também a respeito das complicações que podem surgir, tornando a assistência resolutiva no tempo certo. Sabe-se também, que a equipe de enfermagem tem entre suas responsabilidades, o atendimento integral e descentralizado (MEDEIROS et al., 2015).

A consulta de enfermagem tem papel indispensável tanto para os indivíduos já diagnosticados com diabetes mellitus, como também para aqueles que apesar de não-diabéticos, há uma predisposição ao acometimento dessa doença. Na consulta de enfermagem o profissional consegue conhecer o paciente de maneira holística, conhecendo a sua história pregressa, socioeconômica e até emocional, dessa maneira podendo criar planos de cuidados subjetivos e específicos adequando-se a realidade de cada paciente (Brasil, 2013).

O serviço de enfermagem prioriza a prevenção e promoção da saúde orientado as pessoas a respeito de práticas benéficas para sua qualidade de vida como o uso das medicações, alimentação adequada, atividades físicas, entre outras. É sabido que a enfermagem tem papel fundamental prestando uma assistência integral, holística, humanizada e abrangendo todas as dimensões do sujeito (Barbosa et al., 2014).

\section{Metodologia}

O presente estudo, trata-se de uma revisão bibliográfica de literatura pelo método de revisão integrativa, de caráter qualitativo. De acordo com Pereira et al. (2018), para que o pesquisador apresente seu ponto de vista acerca do estudo, é de suma importância o uso do método qualitativo. Segundo Bento (2012), esse método é indispensável, pois por meio dele adotase um processo sistemático de investigação, que oportuniza agregar conhecimentos sobre a temática estudada, permitindo a identificação de falhas, lacunas e formulação de ideias sobre o tema de estudo por meio da busca de informações sobre a problemática e através de uma análise e interpretação desses resultados.

A verificação foi elaborada por meio das seguintes etapas: 1- Elaboração da pergunta norteadora; 2- Busca nas bases de dados e amostragem; 3- Coleta de dados, 4- Análise críticas dos estudos organizada e organização dos dados (SOUZA, 2010). Para a busca de dados, utilizou-se como fonte de coleta, a plataforma online de pesquisa Biblioteca Virtual em Saúde (BVS) nas bases de dados Literatura Latino Americana e do Caribe em Ciências da Saúde (LILACS), Medical Literature Analysis and Retrieval System Online (MEDLINE), Base de dados em Enfermagem (BDENF) e web artigos, segundo os critérios de inclusão.

Para responder à questão norteadora do estudo: "Qual a produção de enfermeiros sobre as ações que realizam junto aos portadores de diabetes mellitus residentes em Zona Rural, com destaque para os aspectos que consideram relevantes sobre a temática?", utilizou-se o acrônimo PICo, onde, (P=População, I=Interesse, $\mathrm{Co}=\mathrm{Contexto})$. Em que o grupamento "P" referese à população do estudo (diabetes mellitus); "I" à intervenção estudada ou à variável de interesse (enfermagem); "Co" ao contexto de análise da pesquisa (zona rural), a busca dos artigos foi realizada utilizando os Descritores em Ciências da Saúdes (DeCS) e Medical Subject Headings (MeSH) a partir dos operadores booleanos “AND” e "OR”, como descrito no Quadro 1. 
Quadro 1: Quadro de apresentação da estratégia PICo para elaboração da pergunta norteadora e estratégia de busca.

\begin{tabular}{|c|c|c|}
\hline Acrônimos & Componentes & Descs/Machs \\
\hline $\mathbf{P}$ & População & Diabetes mellitus. Diabetes mellitus, Diabetes mellitus \\
\hline $\mathbf{I}$ & $\begin{array}{c}\text { Fenômenos de } \\
\text { interesse }\end{array}$ & Enfermagem, Nursing, Enfermería \\
\hline Co & Contexto & Zona rural, Rural áreas, Medio rural \\
\hline
\end{tabular}

Fonte: Biblioteca Virtual de Saúde (BVS).

A aplicabilidade e arranjo dos descritores da estratégia PICo é de suma importância para o alcance de resultados satisfatórios que atendam aos objetivos desta pesquisa. O que nesta pesquisa resultou na seguinte questão: “Qual a produção de enfermeiros sobre as ações que realizam junto aos portadores de diabetes mellitus residentes em Zona Rural, com destaque para os aspectos que consideram relevantes sobre a temática?".

Foram inclusos apenas artigos publicados nos últimos 05 anos (2017-2021), que estavam acessíveis em textos completos e disponíveis em língua portuguesa, inglesa e espanhola sendo selecionados apenas artigos que corresponderam à temática em estudo. Foram exclusos da consulta todos os trabalhos cuja temática escapava do tema e objetivo apresentado, aqueles que estavam incompletos, que não possuem versão nos idiomas citados, e que foram publicados em período anterior a 2017, repetidos nas bases de dados, e documentos como teses, monografias e dissertações, conforme apresentado na Figura 1.

Figura 1: Fluxograma com resultados das buscas nas bases de dados $(n=10)$.

\begin{tabular}{c} 
Diabetes mellitus.Diabetes mellitus,Diabetes mellitus, \\
Enfermagem,Nursing,Enfermería; \\
Zona rural,Rural áreas,Medio rural \\
\hline
\end{tabular}

Estratégia de busca:((Diabetes mellitus) OR(Diabetes mellitus) OR (Diabetes mellitus)) AND (("Enfermagem")OR ("Nursing") OR ("Enfermería")) AND (("Zona rural") OR ("Rural areas") OR ("Medio rural")) $A N D$ (fulltext:("1" OR "1") AND db:("BDENF"OR "MEDLINE"OR "LILACS") AND mj:("Diabetes mellitus" OR "Papel da enfermagem") $A N D$ la:("en"OR "pt"OR "es")) AND (year_cluster: [2015TO 2020])

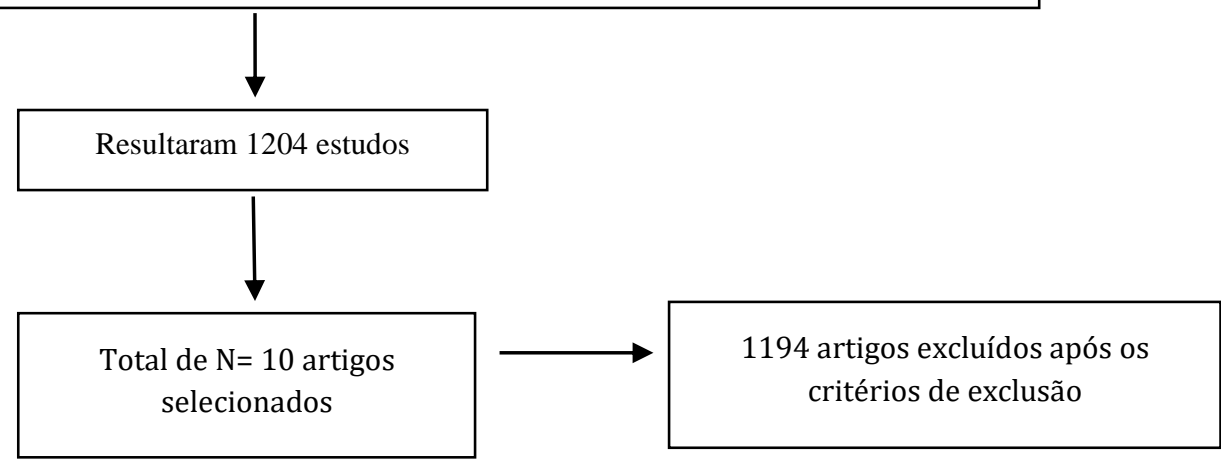

Fonte: Biblioteca Virtual de Saúde. 
O rastreio pelos estudos utilizou-se de dois eixos de informações, no eixo 1: Perfil das produções científicas: Título, ano, autores, idioma, base de dados e abordagem metodológica e no eixo 2- Resultados em evidência, com o questionamento: "Qual a produção de enfermeiros sobre as ações que realizam junto aos portadores de diabetes mellitus residentes em Zona Rural, com destaque para os aspectos que consideram relevantes sobre a temática?”.

\section{Resultados}

Conforme os critérios de inclusão, a partir da leitura e observação do material foram agrupadas as informações importantes a respeito dos estudos encontrados, e estes dados foram organizados em duas figuras: $\mathrm{O}$ Quadro 2 representando o perfil das produções.

Quadro 2: Quadro eixo 1- Perfil das produções (n=10).

\begin{tabular}{|c|c|c|c|c|c|}
\hline $\mathbf{N}^{\mathbf{o}}$ & Título & Ano & Autores & Abordagem & $\begin{array}{c}\text { Base de } \\
\text { dados }\end{array}$ \\
\hline 01 & $\begin{array}{l}\text { Associação entre a prática de atividade física } \\
\text { em diferentes domínios e o uso de insulina } \\
\text { em adultos e idosos com diabetes no brasil. }\end{array}$ & 2020 & STREB et al & Quantitativo & SCIELO \\
\hline 02 & $\begin{array}{l}\text { Uso dos serviços de saúde segundo } \\
\text { determinantes sociais, comportamentos em } \\
\text { saúde e qualidade de vida entre diabéticos }\end{array}$ & 2020 & $\begin{array}{l}\text { RODRIGUES } \\
\text { et al }\end{array}$ & Quantitativo & SCIELO \\
\hline 03 & $\begin{array}{l}\text { Abordagens metodológicas utilizadas em } \\
\text { intervenções educativas voltadas a } \\
\text { indivíduos com diabetes mellitus. }\end{array}$ & 2020 & $\begin{array}{l}\text { SANTOS, } \\
\text { Wallisson }\end{array}$ & Qualitativo & SCIELO \\
\hline 04 & $\begin{array}{l}\text { Fatores relacionados ao diabetes mellitus que } \\
\text { podem influenciar no autocuidado. }\end{array}$ & 2020 & SOUZA et al. & Quantitativo & LILACS \\
\hline 05 & $\begin{array}{l}\text { Adesão ao tratamento de diabetes mellitus e } \\
\text { relação com a assistência na atenção } \\
\text { primária }\end{array}$ & 2020 & $\begin{array}{l}\text { SANTOS et } \\
\text { al. }\end{array}$ & Quantitativo & BDENF \\
\hline 06 & $\begin{array}{l}\text { Conhecimento e atitude sobre diabetes } \\
\text { mellitus de usuários idosos com a doença } \\
\text { atendidos em unidade básica de saúde }\end{array}$ & 2020 & $\begin{array}{l}\text { GONÇALVE } \\
\text { S et al. }\end{array}$ & Quantitativo & BDENF \\
\hline 07 & $\begin{array}{l}\text { Qualidade de vida relacionada à saúde de } \\
\text { pacientes com diabetes mellitus }\end{array}$ & 2020 & $\begin{array}{l}\text { MARQUES et } \\
\text { al. }\end{array}$ & Quantitativo & BDENF \\
\hline 08 & $\begin{array}{l}\text { Protocolo de enfermagem para o cuidado da } \\
\text { pessoa com diabetes mellitus na atenção } \\
\text { primária }\end{array}$ & 2020 & $\begin{array}{l}\text { LAUTERTE } \\
\text { et al. }\end{array}$ & Qualitativo & BDENF \\
\hline 09 & $\begin{array}{l}\text { A hospitalização como espaço para educação } \\
\text { em saúde às pessoas com diabetes mellitus }\end{array}$ & 2020 & $\begin{array}{l}\text { ARRUDA e } \\
\text { SILVA. }\end{array}$ & Qualitativo & BDENF \\
\hline 10 & $\begin{array}{l}\text { Diabetes mellitus: estratégias de educação } \\
\text { em saúde para o autocuidado }\end{array}$ & 2021 & $\begin{array}{l}\text { BREHMER et } \\
\text { al. }\end{array}$ & Qualitativo & BDENF \\
\hline
\end{tabular}

Fonte: Biblioteca Virtual de Saúde (BVS).

Como exposto na figura anterior, observa-se a maioria dos estudos com ano de publicação 2020 totalizando 9 artigos, apresentando apenas 1 estudo do ano de 2021. No que se refere aos idiomas empregados, não foram selecionados artigos nos idiomas inglês e espanhol, sendo a língua portuguesa a predominante, empregada em todos os artigos. Na abordagem metodológica são 6 estudos quantitativos e apenas 4 qualitativos. Vale salientar a diversidade de periódicos, em que houve o emprego maior na base de dados em Enfermagem (BDENF), com 7 publicações, seguida da scientific electronic library (SCIELO), com 3 publicações restantes.

Já o Quadro 3 aborda os resultados em evidência retirados de cada publicação após uma análise criteriosa, feita por dois revisores separadamente, e enviadas a um terceiro, a fim de responder o seguinte questionamento: "Qual a produção de 
enfermeiros sobre as ações que realizam junto aos portadores de diabetes mellitus residentes em Zona Rural, com destaque para os aspectos que consideram relevantes sobre a temática?"

Quadro 3: Quadro com os Resultados em evidência ( $\mathrm{n}=10)$.

\begin{tabular}{|c|c|c|}
\hline $\mathbf{N}^{\mathbf{o}}$ & Título & $\begin{array}{l}\text { Qual a produção de enfermeiros sobre as ações que realizam junto aos portadores } \\
\text { de diabetes mellitus residentes em Zona Rural, com destaque para os aspectos que } \\
\text { consideram relevantes sobre a temática? }\end{array}$ \\
\hline 01 & $\begin{array}{l}\text { Associação entre a prática de } \\
\text { atividade física em diferentes } \\
\text { domínios e o uso de insulina em } \\
\text { adultos e idosos com diabetes no } \\
\text { brasil. }\end{array}$ & $\begin{array}{l}\text { O estudo apontou que a realização de atividades físicas na residência e nos ambientes de } \\
\text { trabalho reduzem a necessidade da utilização de insulina como tratamento para diabetes } \\
\text { em idosos. Apresentando percentuais de redução à medida que se agreguem á prática } \\
\text { dessas atividades em mais ambientes. }\end{array}$ \\
\hline 02 & $\begin{array}{l}\text { Uso dos serviços de saúde } \\
\text { segundo determinantes sociais, } \\
\text { comportamentos em saúde e } \\
\text { qualidade de vida entre diabéticos }\end{array}$ & $\begin{array}{l}\text { O estudo sugere que diferenças relacionadas aos determinantes sociais e o impacto do } \\
\text { diabetes na qualidade de vida podem explicar a utilização dos serviços de saúde. Ações } \\
\text { voltadas para a promoção do bem-estar, saúde, prática de autocuidado e qualidade de } \\
\text { vida entre os diabéticos devem ser realizados de maneira constante dentro das ESF. }\end{array}$ \\
\hline 03 & $\begin{array}{l}\text { Abordagens metodológicas } \\
\text { utilizadas em intervenções } \\
\text { educativas voltadas a indivíduos } \\
\text { com diabetes mellitus. }\end{array}$ & $\begin{array}{l}\text { O estudo apontou que existe uma necessidade de investigação dentro do tema e que } \\
\text { contemplem diversas realidades e limitações das estratégias, tal como adaptações ou } \\
\text { elaboração de outras ferramentas que sejam fundamentadas em evidências científicas, } \\
\text { atestando a sua efetividade junto à prática clínica. }\end{array}$ \\
\hline 04 & $\begin{array}{l}\text { Fatores relacionados ao diabetes } \\
\text { mellitus que podem influenciar no } \\
\text { autocuidado. }\end{array}$ & $\begin{array}{l}\text { Este estudo destaca a relevância do profissional enfermeiro na assistência ao paciente } \\
\text { diabético residente em zona rural, sendo por meio da consulta de enfermagem e/ou } \\
\text { assistência que obtém-se o conhecimento de sua história pregressa e socioeconômica, } \\
\text { hábitos, assim como criar planos de cuidados específicos para cada paciente de acordo } \\
\text { com suas características, capacitando-os por meio de orientações de autocuidado e } \\
\text { estimulando a ajuda da família aos mesmos. }\end{array}$ \\
\hline 05 & $\begin{array}{l}\text { Adesão ao tratamento de diabetes } \\
\text { mellitus e relação com a } \\
\text { assistência na atenção primária }\end{array}$ & $\begin{array}{l}\text { O estudo mostra que os pacientes acometidos pela DM2 apresentam boa adesão ao } \\
\text { tratamento medicamentoso, porém no que se refere ao tratamento não-medicamentoso, o } \\
\text { que aponta a necessidade de que as equipes da ESF precisam ampliar a implementação } \\
\text { de ações de promoção da saúde, prevenção e controle da doença e suas complicações. }\end{array}$ \\
\hline 06 & $\begin{array}{l}\text { Conhecimento e atitude sobre } \\
\text { diabetes mellitus de usuários } \\
\text { idosos com a doença atendidos } \\
\text { em unidade básica de saúde }\end{array}$ & $\begin{array}{l}\text { Este estudo mostra que a adesão do tratamento entre os idosos e o aumento das taxas de } \\
\text { complicações associadas ao DM estão diretamente ligados ao baixo nível de } \\
\text { conhecimento e atitude negativa em relação ao DM. }\end{array}$ \\
\hline 07 & $\begin{array}{l}\text { Qualidade de vida relacionada à } \\
\text { saúde de pacientes com diabetes } \\
\text { mellitus }\end{array}$ & $\begin{array}{l}\text { O estudo mostra que a maioria dos pacientes não se sentem constrangidos com relação a } \\
\text { sua doença, porém preocupam-se com possíveis complicações relacionadas a ela, o que } \\
\text { deixa evidente a importância das ações de enfermagem também no âmbito da pesquisa } \\
\text { cientifica relacionada às perspectivas dos pacientes. }\end{array}$ \\
\hline 08 & $\begin{array}{l}\text { Protocolo de enfermagem para o } \\
\text { cuidado da pessoa com diabetes } \\
\text { mellitus na atenção primária }\end{array}$ & $\begin{array}{l}\text { Este estudo destaca a importância do protocolo de enfermagem para o cuidado da pessoa } \\
\text { com diabetes mellitus na atenção primária na relação à atuação do enfermeiro, o mesmo } \\
\text { possibilitou autonomia profissional e respaldo para execuçâo dos princípios e diretrizes } \\
\text { da Política Nacional de Atenção Básica, tais como o fortalecimento do vínculo, } \\
\text { longitudinal idade, coordenação do cuidado, resolutividade e melhoria da acessibilidade, } \\
\text { por meio da ampliação da clínica. }\end{array}$ \\
\hline 09 & $\begin{array}{l}\text { A hospitalização como espaço } \\
\text { para educação em saúde às } \\
\text { pessoas com diabetes mellitus }\end{array}$ & $\begin{array}{l}\text { O estudo aponta o protagonismo do profissional enfermeiro como agente de mudanças } \\
\text { ao se posicionar em uma atuação proativa para assim quebrar barreiras e superar } \\
\text { obstáculos, desenvolvendo ações em todos os âmbitos até mesmo durante a } \\
\text { hospitalização decorrente de complicações associadas a DM. }\end{array}$ \\
\hline 10 & $\begin{array}{l}\text { Diabetes mellitus: estratégias de } \\
\text { educação em saúde para o } \\
\text { autocuidado }\end{array}$ & $\begin{array}{l}\text { O estudo ressalta a importância da utilização das mídias sociais nas ações de saúde para } \\
\text { o alcance de um maior número de pessoas, assim beneficiando um maior número de } \\
\text { indivíduos }\end{array}$ \\
\hline
\end{tabular}

Fonte: Microsoft Office Excel (2019). 
Analisou-se que todos os 10 artigos trabalhavam assiduamente a importância das ações de enfermagem em benefício dos portadores de DM, principalmente aqueles que residem em ambiente rural por conta dos desafios enfrentados por eles e as suas particularidades com relação a aspectos como a adesão do tratamento e autocuidado.

\section{Discussão}

Streb et al (2020), realizaram um estudo analisando dados do inquérito Vigilância de fatores de risco e proteção para doenças crônicas por Inquérito Telefônico, objetivando investigar a associação entre o uso de insulina e a realização de atividades físicas. A pesquisa demonstrou que a prática de atividades físicas no domicílio e durante o trabalho têm como resultado a diminuição das chances de necessitar da utilização de insulina como tratamento para o diabetes em idosos e pessoas em idade adulta. Ressaltando que, quanto mais domínio a atividade física for empregada, maior a redução das chances desses diabéticos precisarem da utilização de insulina, ou seja, o emprego dessas atividades é indispensável para diabéticos tanto em rotinas urbanas quanto rurais.

Em outro estudo Rodrigues et al (2020), ao realizarem investigação a respeito da utilização dos serviços de saúde de acordo com determinantes sociais e qualidade de vida entre os diabéticos sugerem que as diferenças ligadas aos determinantes sociais, o efeito do diabetes na qualidade de vida dos usuários e os comportamentos em saúde explicam a utilização dos serviços de saúde. Além disso, evidencia a importância da realização contínua de ações que tenham como finalidade principal promover a qualidade de vida, o bem-estar, o autocuidado e principalmente a saúde entre os diabéticos.

Em outra abordagem, Santos (2020), identificou abordagens metodológicas que são usadas nas ações educativas direcionadas para diabéticos destaca a importância de pautar as intervenções em técnicas dinâmicas e interativas que busquem interatividade com o público alvo permitindo que estes possam ressignificar a sua rotina, estilo de vida e saúde, favorecendo tanto uma melhor convivência com a doença crônica como conhecimentos a respeito de como agir em determinadas situações e /ou complicações que possam acontecer, assim evitando maiores consequências decorrentes destes acontecimentos que podem vir a decorrer.

A pesquisa desenvolvida por Souza et al (2020), analisou os tipos de autocuidado realizados por portadores de diabetes mellitus acompanhados em uma ESF da zona rural, identificando que os pacientes seguem a terapia medicamentosa, porém não mudaram de maneira ativa seus hábitos de vida e saúde, o que favorece agravos à doença. Ressaltando a relevância do profissional enfermeiro, uma vez que é este que por meio da consulta e/ou assistência de enfermagem irá obter um conhecimento mais aprofundado e holístico a respeito daquele paciente, podendo assim desenvolver planos de cuidados específicos para cada paciente, estimulando o autocuidado e mudança nos hábitos de vida por meio da autonomia proporcionada pelos conhecimentos repassados ao paciente.

O artigo de Santos et al (2020), analisou tanto a adesão ao tratamento medicamentoso e não medicamentoso como as ações prestadas aos portadores de DM por equipes da Estratégia da Saúde da Família. Constando boa a adesão ao tratamento medicamentoso e em contrapartida baixa adesão ao tratamento não medicamentoso ao detectar fatores como a ausência de atividades físicas e má alimentação. Contudo a adesão ao tratamento está diretamente relacionada à assistência prestada, o que reforça que a equipe de saúde tem papel indispensável na assistência ao diabético por meio de ações de promoção, prevenção e controle tanto da doença como de possíveis complicações na esfera individual e coletiva.

Gonçalves et al (2020), abordaram o conhecimento de idosos portadores de DM a respeito da própria doença, detectando baixo nível de conhecimento o que denota baixo empenho na adesão do tratamento, tendo como consequência a elevação das taxas de complicações relacionadas a doença. O que propões a execução de ações educativas voltadas para este público com o objetivo de elevar os níveis de conhecimentos destes diabéticos reduzindo assim as consequências negativas de um nível raso de entendimento. 
Marques et al (2020), ao investigar a qualidade de vida de pacientes acometidos pela DM2 observou que a maioria dos pacientes diabéticos relatam estar médio satisfeitos com a sua qualidade de vida em geral e que estes têm muitas preocupações com relação a possíveis complicações. Com isso foi concluído que estes elementos são de suma importância para subsidiar uma assistência completa, indo além da visão clínica em si.

Lauterte et al (2020), ressalta a contribuição do protocolo de enfermagem para o desenvolvimento do cuidado aos pacientes diagnosticados com DM para as equipes de saúde da Atenção Primária. Constatando a importância do estudo do protocolo pelos enfermeiros, a fim de aprimorar o cuidado e a transferência de conhecimento aos pacientes.

O estudo de Arruda e Silva (2020), buscaram compreender como a equipe de enfermagem desenvolve os cuidados aos pacientes diagnosticados com diabetes mellitus por meio da educação em saúde dentro do ambiente hospitalar. E dentre os achados foi possível evidenciar a relevância da promoção de educação em saúde no cenário hospitalar no cotidiano dos pacientes, uma vez que é possível traçar estratégias para a aplicabilidade destas ações abordando pacientes, cuidadores e/ou familiares.

Por fim, Brehmer et al (2021), descrevem as principais estratégias realizadas em ações de extensões voltadas para o DM. Destacando que as ações educativas desenvolvidas pelo grupo proporcionaram melhora significativa na qualidade de vida e autonomia dos diabéticos, o que ressalta a indispensabilidade da aplicação de ações em saúde para o desenvolvimento de autocuidado e conhecimento eficaz do diabético.

\section{Conclusão}

Os estudos analisados demonstraram que a aplicabilidade e aperfeiçoamento das ações que podem ser desenvolvidas pelo profissional enfermeiro são de total relevância tanto para a prevenção como para a lida com a doença depois de diagnosticada, principalmente no cenário rural onde ficou evidenciada a necessidade de um olhar mais especial para estes.

Pode-se observar que a maioria dos estudos investigados enfatiza o autocuidado como primordial para o paciente que convive com algum dos tipos de DM, fazendo-nos refletir sobre a assistência que deve ser prestada à aqueles que vivem em ambientes rurais, evidenciando que é preciso ter um olhar holístico a fim de desenvolver cuidados que se adequem às sua realidade como um todo envolvendo todos os aspectos necessários para transferir todo o conhecimento necessário para formar diabéticos com qualidade de vida e saúde, praticando o autocuidado com autonomia e segurança.

Diante dos achados que confirmam a importâncias das ações de enfermagem realizadas para os diabéticos que residem em ambiente rural, sugere-se que haja o desenvolvimento de estudos com a temática em questão para que o debate a respeito destas ações que por sua vez são primordiais para a promoção da qualidade de vida, autonomia, autocuidado, prevenção e habilidade diante de possíveis complicações, seja mais fortalecida para assim estimular uma assistência humana, completa e com um olhar sensível àqueles que estão em ambientes que requerem um cuidado mais especifico como os residentes da zona rural.

\section{Referências}

Arruda, C., \& Silva, D. M. G. V. da. (2020). La hospitalización como espacio para la educación en salud a las personas con diabetes mellitus. Revista De Pesquisa: Cuidado é Fundamental, 12(1. http://ciberindex.com/c/ps/P037045.

Brasil \& Ministério da Saúde. (2013). Estratégias para o cuidado da pessoa com doença crônica: diabetes mellitus. Cadernos da Ateção Basica, . 36.

Brehmer, L. C. F., Canever, B. P., Rosa, L. M., Locks, M. O. H., Manfrini, G. C., \& Willrich, G. P. B. (2021). Diabetes Mellitus: estratégias de educação em saúde para o autocuidado. Rev. enferm. UFPE on line. 1-16.

Cotta, R. M. M., Batista, S. C., Reis, R. S., Souza, A. G., Dias, G., Castro, F. A. F., \& Alfeas, R. C. G. (2009). Perfil sócios sanitário e estilo de vida de hipertensos e/ou diabéticos, usuários do Programa de Saúde da Família no município de Teixeiras. Rev. Ciênc. saúde coletiva. 
Farias, R. F. S., Lima, A. W. S., Leite, A. F. B., Santos, Z. C., Santos. E. C., \& Dias, A. A. (2017). Adesão ao tratamento de diabetes mellitus em área rural do município de vitória de santo antão - pe. Revista de APS. 181 - 190.

Farinha, F. T., Oliveira, B. N. D., Santos, S. F. C., Souza, W. R., Razera, A. P. R., \& Trettene, A. S. (2020). Atividades de autocuidado em pacientes com Diabetes Mellitus tipo 2: estudo transversal. Rev. enferm. UERJ . 1-7.

Gonçalves, L. H. T., Silva, A. P., Fernandes, D. S., Carlos, L. F., Castro, R. L. P., \& Uchôa, V. S. (2020). Conhecimento e atitude sobre o diabetes mellitus de usuários idosos coma doença atendidos em unidade básica de saúde. Nursing (Säo Paulo). 3497-3501.

Lima, W. R., Giusti, B. B., Niwa, L. M. S., Aanholt, D. P. J., \& Ciosak, S. I. (2021). Idosos muito velhos: perfil sociodemográfico, de saúde e longevidade. Rev. enferm. UFPE on line. 15(1).

Lauterte, P., Silva, D. M. V. G., Salci, M. A., Heidemann, I. T. S. B., \& Romanoski, P. J. (2020). Protocolo de enfermagem para o cuidado da pessoa com diabetes mellitus na atenção primária. Rev. enferm. UFSM ; 10: 72

Marques, J. V. P., Teixeira, C. R. S., Zanetti, M. L., Kusumota, L., Becker, T. A. C., \& Hodniki, P. P. (2020). Qualidade de vida relacionada à saúde de pacientes com Diabetes Mellitus. Rev. Pesqui. (Univ. Fed. Estado Rio J., Online) ; 12: 1050-1058

Pereira, S. W., (2020). Enfoques metodológicos utilizados en intervenciones educativas dirigidas a personas con diabetes mellitus / Methodological approaches used in educational interventions aimed at individuals with diabetes mellitus. Enferm. actual Costa Rica (Online) ; (38): 260-271

Rodrigues, A. M. A. M., Cavalcanti, A. L., Pereira, J. L. S. H., Araujo, C. L. C., Beradio, I. M., Soares, R. L., Freire, D. E. W. G., \& Soares, R. S. C. (2020). Uso dos serviços de saúde segundo determinantes sociais, comportamentos em saúde e qualidade de vida entre diabéticos. Cien Saude Colet ; 25(3): 845-858

Santos, A. L., Marcon, S. S., Teston, E. F., Black, I. R., Lino, I. G. T., Batista, V. C., Matsuda, L. M., \& Haddad, M. C. F. L. (2020). Adesão ao tratamento de diabetes mellitus e relação com a assistência na atenção primaria. REME rev. min. enferm .1-10.

Schroder, A. C., Vanz, A. P., Geremia, C., Trindade, C. S., \& Canabarro, S. T. (2021). Telessaúde em um centro de referência em Diabetes Mellitus: uma análise transversal. Esc. Anna Nery. 1414-8145.

Serra, E. B., Ferreira, A. G. N., Pascoal, L. M., \& Rolim, I. L. T. P. (2020). Diagnósticos de enfermagem em pacientes diabéticos: revisão integrativa. Rev. enferm. UERJ. 1-9.

Streb, A.R., Leonel, L. D. S., Silva, C. S., Silva, R. P., \& Duca, G. F. D. (2020). Associação entre a prática de atividade física em diferentes domínios e o uso de insulina em adultos e idosos com diabetes no Brasil. Cien Saude Colet. 4615-4622.

Souza, N. M. S., Cunha, A. C., Rezende, F. M., Quadros, K. A. N., Santos, R. C., \& Andrade, S. N. (2020). Fatores relacionados ao diabetes mellitus que podem influenciar no autocuidado. Nursing (Säo Paulo). 4580-4588.

Sousa, W. E. A., Sardinha, A. H. L., Verzaro, P. M., Balata, I. L. B., Santos, S. R., \& Guterres, D. M. B. (2021). Funcionalidade familiar de idosos com diabetes mellitus. Rev. Pesqui. (Univ. Fed. Estado Rio J., Online), 612-618.

Valentim, S. A., Haddad, M. C. L., \& Rossaneis, M. A., Dificuldades vivenciadas pelo portador de diabetes mellitus residente em distrito rural. Rev Enferm UFPE online.

Zanetti, M. L., Cordeiro, A. A C., Franco, R. C. S., Manoel, A. L. R, \& Turcatto, G. F. L. (2015). Adesão às recomendações nutricionais e variáveis sociodemográficas em pacientes com diabetes mellitus. Revista da Escola de Enfermagem da USP (São Paulo), 619-625. 